\title{
Reinigungsbranche quo vadis
}

\author{
Nach einem eher durchwachsenen Jahr 2019 starteten einige Hersteller von Reinigungsanlagen \\ hoffnungsfroh ins neue Jahrzehnt. Mit dem weltweiten Stopp brachen die Auftragseingänge \\ jedoch drastisch ein. Inzwischen stabilisiert sich die Situation wieder etwas. Trotzdem: Eine \\ Marktbereinigung schließt die Branche nicht aus.
}

\begin{abstract}
Aufträge aus dem Boomjahr 2018 sorgten teilweise bis weit ins Jahr 2019 für eine gut ausgelastete Produktion bei den Anlagenherstellern. Durch die mehr oder weniger massiven Polster war es noch verschmerzbar, dass der Auftragseingang in der zweiten Hälfte des letzten Jahres zum Teil schon deutlich eingebrochen ist - insbesondere Bestellungen aus der Automobilindustrie und von deren Zulieferern. Der bei den meisten Herstellern vorhandene Auftragsbestand zum Jahresende und ein für manchen Anlagenbauer guter bis sehr guter Start ins Jahr 2020 machten Hoffnung.
\end{abstract}

\section{Gute Aussichten ausgebremst}

Die Automobilindustrie und ihre Zulieferer kränkeln zwar schon länger, aber aus anderen Industriebereichen wie der Medizintechnik, dem Halbleiter-Zulieferbereich, der Laser- und Sensortechnik, dem allgemeinen Getriebe- und Sondermaschinenbau, der Hydraulik oder auch der additiven Fertigung kamen Aufträge. Sie sorgten bei den meisten Anlagenherstellern für einen positiven Ausblick auf das gerade angelaufene Jahr.

Dann kam im März der große Stopp über alle Branchen hinweg. Ein großer Teil der geplanten Projekte wurden von Kunden auf „hold“ gestellt und Aufträge verschoben. Zur Stornierung von Aufträgen kam es bei keinem der Anlagenhersteller.

In dieser Situation half es nur bedingt, dass China und andere asiatische Länder ab April wieder etwas aktiver wurden. Schwierigkeiten vorhandene Aufträge zu realisieren hatten die Anlagenhersteller teilweise aufgrund unterbrochener Lieferketten. Alle befragten Anlagenbauer nutzten die Möglichkeit der Kurzarbeit erstmals in deren Unternehmensgeschichte auch im Vertrieb. Daraus resultierten Auftragseinbußen, die im Vergleich zu den ersten sechs Monaten 2019 zwischen 25 und 50 \% lagen, nur ein Unternehmen berichtete von einem etwas höheren Auftragseingang als im ersten Halbjahr 2019.

\section{Marktbereinigung erwartet}

Darüber hinaus führten und führen die Kontaktbeschränkungen und für bestimmte Länder noch vorhandene Reiserestriktionen $\mathrm{zu}$ Verzögerungen bei der Auslieferung fertiggestellter Anlagen. Entsprechend bleiben eingeplante Zahlungen aus, was für die Hersteller der Reinigungssysteme eine weitere Herausforderung darstellt. Gleichzeitig verschärfen sich bei Aufträgen aus den klassischen Anwenderbranchen, die momentan vergeben werden, die Preisverhandlungen. Die durch Corona ausgelöste Krise wird von der Branche im Vergleich zur Finanzkrise 2008/2009 als tiefgreifender bewertet. Sie geht davon aus, dass eine gewisse Marktbereinigung stattfinden wird.

\section{Absehbare Entwicklungen und internationale Hürden}

Dazu trägt auch der tiefgreifende Strukturwandel in der Automobilindustrie bei, die mit ihren Zulieferern nach wie vor die Schlüsselbranche für Anbieter von Reinigungstechnik ist. Der über Jahre durch Kapazitätserweiterungen getriebene An- lagenbedarf für Komponenten von Verbrennungsmotoren hat sich bereits seit Mitte 2018 abgeschwächt. Der globale Absatzeinbruch und die über Wochen stillstehenden Produktionen bei den OEM wird diese Entwicklung noch verstärken. Sichtbar wird dies bereits durch Insolvenzen in der Automobil-Zulieferbranche. Hemmnisse bestehen auch auf internationaler Bühne. So kommt es für die exportorientierten Anlagenbauer darauf an, wie schnell und gut die Industrie in anderen Volkswirtschaften wieder auf die Beine kommen wird. Ebenso die Frage, ob es nach dem vollzogenen Brexit ein Handelsabkommen zwischen der EU und Großbritannien geben wird. Darüber hinaus richtet sich der Blick auf die Präsidentschaftswahl in den USA: Bleibt Donald Trump an der Macht und kann seine protektionistische Zollpolitik fortsetzen?

\section{Geduld und Diversifizierung}

Inzwischen haben Auftragseingänge und die Projekttätigkeit bei den meisten Anlagenbauern wieder mehr oder weniger stark angezogen. Beratungen, Verhandlungen und Versuche erfolgen dabei teilweise noch per Videokonferenz. Einigkeit besteht jedoch darüber, dass ein zweiter Lockdown dramatische Folgen haben würde. Und auch, dass es einige Zeit dauern wird, bis die Volumen der guten Jahre wieder erreicht werden. Eine Voraussetzung dafür ist eine stärkere Diversifizierung in neue Branchen und Marktregionen, um dadurch Abhängigkeiten von der Automobilindustrie deutlich zu verringern. //

Doris Schulz, Fachjournalistin, Stuttgart 